\title{
A rectangular slot Yagi DGS microstrip filter with sharp rolloff and wide suppression band
}

\author{
Jing Gong ${ }^{1}$
}

\begin{abstract}
A compact microwave lowpass filter based on defected ground structure (DGS), consisting of Yagi slot DGS resonators etched on the metallic ground plane of a microstrip line, is studied in this paper. The proposed lowpass filter was analyzed by parameter sweeping and optimal parameters were selected to design a prototype demonstrator filter. Measurements show the developed lowpass filter exhibits a $3 \mathrm{~dB}$ cutoff frequency of $3.07 \mathrm{GHz}$ with a sharp transition between the passband and the stopband, low insertion loss of less than $0.6 \mathrm{~dB}$ in the passband and an in-band group delay of $0.4 \mathrm{~ns}$. Moreover, an ultra-wide suppression band from 3.68 to over $20 \mathrm{GHz}$ with a suppression level generally better than $20 \mathrm{~dB}$ is also observed from measurements.

K e y w o r d s: defected ground structure (DGS), microwave lowpass filter, suppression band, microstrip line
\end{abstract}

\section{Introduction}

The rapid development in telecommunication systems and applications demands compact, low profile and miniaturized microwave components for application to such as satellite systems, mobile communications, wireless remote sensing, super resolution transmissions and imaging [1], and so on. In all the above mentioned applications, microwave components such as diplexers [2], [3], antennas [4]-[6], baluns [7], [8], power dividers [9][12] and filters [13], [14] may be used in one way or the other. Among them, the microwave filters are indispensable in many RF and microwave applications and they may function as separating or combining signals of different frequencies as a result of the electromagnetic spectrum being a limited resource that needs to be shared. For this purpose, microwave filters are necessary to confine or select microwave or radio frequency signals within dedicated spectral limits. This calls for filters with low cost, small size, low profile, ultra-wide suppression band, sharp rolloff from the passband to stopband, minimal in-band insertion loss and so on.

In 1957, Hamel and Isbell proposed the conventional log-periodic array antenna for broadband applications [15], [16] and in 1977, Campbell et al presented the printed log-periodic dipole array antenna [17]. The logperiodic structures have found wide applications in antenna designs and was introduced into the design of microwave circuits. In [18], authors proposed a compact logperiodic branch-line balun with an octave bandwidth and a compact size. In [19], authors proposed a quasi-Yagi defected ground structure (DGS) lowpass filter with a sharp cutoff frequency response and low in-band insertion loss along with a wide rejection band of about $20 \mathrm{~dB}$ from 2.8 to $11 \mathrm{GHz}$, where a further size reduction is required.
The DGS resonator for microwave filter applications has become prevalent due to its inherent bandgap properties over photonic bandgap structures at some frequencies that exhibits finite attenuation poles. On the other hand, the design of lowpass filters with ultra-wide suppression band is a daunting task and no wonder earlier reported works such as the dumb-bell-shaped DGS had a narrow suppression band. To mitigate these challenges, DGS unit cells may be cascaded to obtain a wide suppression band at the expense of circuit size and increases the in-band insertion loss that may be undesired for compact applications. In [20], cross-shaped DGS lowpass filter was designed at a cutoff frequency of $4 \mathrm{GHz}$ with an ultra-wide suppression band from 4.3 to $20 \mathrm{GHz}$ referred to $20 \mathrm{~dB}$. In [21], fan-shaped DGS and double radial stub was used to implement a lowpass filter with a cut-off frequency at 1.49 $\mathrm{GHz}$ while has the first attenuation pole at $2.0 \mathrm{GHz}$ with an attenuation level of $57.2 \mathrm{~dB}$. The developed filter has a stopband range from 1.65 to $7.41 \mathrm{GHz}$ and a suppression level of $20 \mathrm{~dB}$ but a bulky size. These results illustrate developing microwave filters with high performance and compact size/volume would be always challenging.

In this study, a microstrip Yagi slot DGS lowpass filter at a $3 \mathrm{~dB}$ cutoff frequency of $3.07 \mathrm{GHz}$ is developed with a low profile and compact size as well as an ultra-wide suppression band from 3.68 to over $20 \mathrm{GHz}$ under a 20 $\mathrm{dB}$ suppression level. This is a Yagi DGS resonator for application to developing the microwave lowpass filters. The design and development of such a microwave filter is presented and discussed with the proposed parameter sweeping. And, subsequently, a simulation study is carried out.

\footnotetext{
${ }^{1}$ Chengxian College, Southeast University, Nanjing 210088, China, magi_gj@163.com, magi_gj@126.com
} 


\section{Rectangular slot based Yagi DGS resonator}

The concept and principle of the log periodic array antenna have been introduced as a DGS in microstrip lowpass filter design to realize a Yagi DGS microstrip lowpass filter. It is composed of individual scaled rectangular slots to form the lowpass filtering on a microwave grounded substrate. The log periodic etched slots on the ground plane act as resonators for the lowpass filter implementation, which distracts the shield current flow on the ground plane. It is evident from [15] that for structures comprising of resonant elements, if the frequency independent performance is expected, the resonances must be staggered to enable the variation of frequency in relation to the resonant element from one to another. For the case of antenna array composed of similar discrete elements, it implies that the physical sizes of the elements must be scaled from one to the next such that the desired frequency range can be covered with elements of overlapping response characteristics.

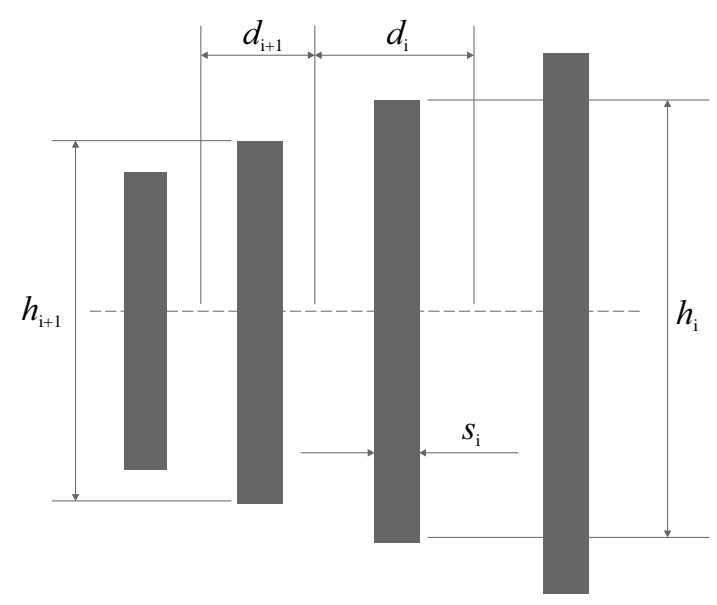

Fig. 1. Rectangular slot based YAGI DGS

The concepts formulated above have been adopted to design microstrip DGS lowpass filters for microwave applications. Scaled defected slots arranged side by side have been etched out on the metallic ground plane below a microstrip line sandwiched by a microwave substrate. Fig. 1 shows the studied Yagi DGS resonator. It consists of rectangular slots scaled in height (length) and separation to represent a log periodic pattern etched on the ground plane of a $50 \Omega$ microstrip line. The periodic marbled slots on the metallic ground plane disrupts the current distributions, causing modifications of the transmission line characteristics and thus creating the slowwave effect and realizing the band rejection property [14]. When a half wavelength slot resonates, consequently it controls the center frequency of its transmission and reflection responses when applied to microwave filter designs. In general, the number of scaled rectangular slots, their scaled length and separation with respect to the 50 $\Omega$ microstrip line as well as the substrate determine the filter responses. Here we represent the width of the rectangular slot as $s_{i}$, while the height (length) of the slot is denoted as $h_{i}$ and the separation between adjacent slots is described as $d_{i}$. Further, we define the height (length) scale factor, $R_{\mathrm{h}}$, as

$$
R_{\mathrm{h}}=\frac{h_{i+1}}{h_{i}}, \quad i=1,2,3, \ldots
$$

The lengths of the defected rectangular slots forming the Yagi DGS lowpass filter are related by a constant scale factor denoted as $R_{\mathrm{h}}$ in (1). For the operation of this type of structure, it is also necessary to scale the environment, namely the separation between adjacent defected slots must have a relation in like manner. Hence the separation ratio between rectangular slots is defined as

$$
R_{\mathrm{d}}=\frac{d_{i+1}}{d_{i}}, \quad i=1,2,3, \ldots
$$

On the other hand, to present a more general discussion about such a kind of DGS, the slot width (representing the characteristic impedance of a slotline) is also varied with a ratio given by

$$
R_{0} \mathrm{~s}=\frac{s_{i+1}}{s_{i}}, \quad i=1,2,3, \ldots
$$

It is noted that the microwave substrate in this study has a relative permittivity of 9.6 with a thickness of 0.8 $\mathrm{mm}$.

\section{Developing microstrip DGS filter based on Yagi slot resonators}

The 3D view of the discussed Yagi DGS lowpass filter is shown in Fig. 2, where seven basic rectangular DGS cells were cascaded in a Yagi fashion based on the architecture shown in Fig. 1. On the top side of the grounded substrate, it is a $50 \Omega$ microstrip line with its line width $0.76 \mathrm{~mm}$ for the above mentioned microwave substrate. The Yagi DGS filter shown in Fig. 2 is numerically analyzed by parameter sweeping. A full-wave EM simulator, Ansoft Ensemble, was employed to carry out the analyses.

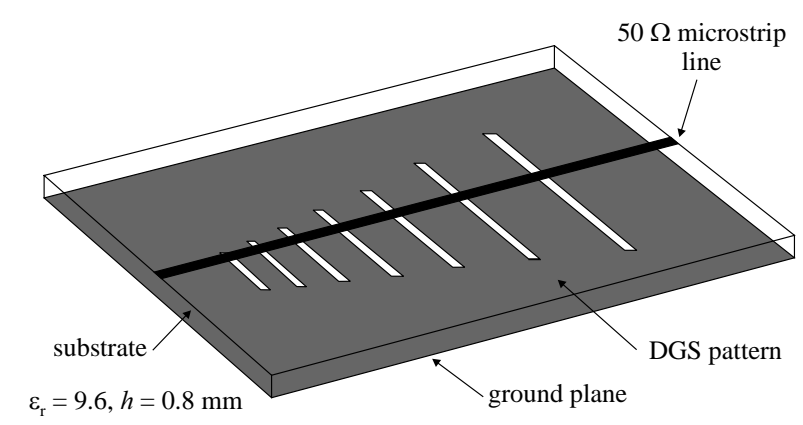

Fig. 2. 3D view of the proposed Yagi DGS microstrip filter 


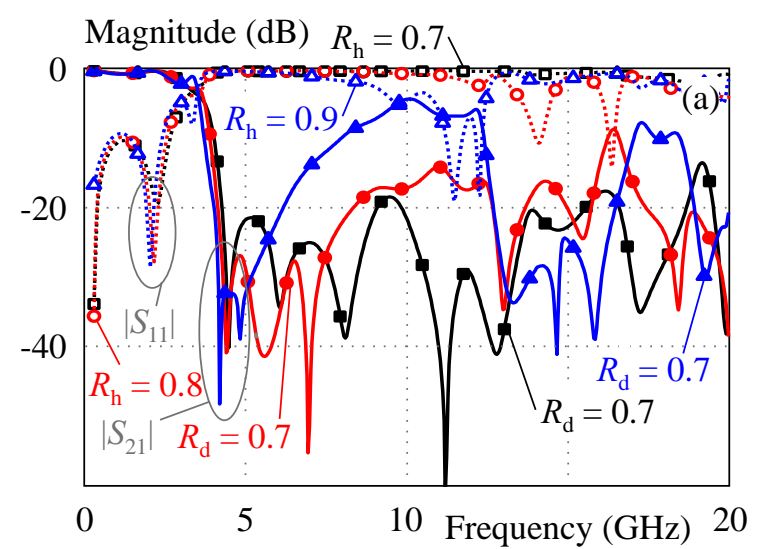

Magnitude (dB) $R_{\mathrm{h}}=0.7$

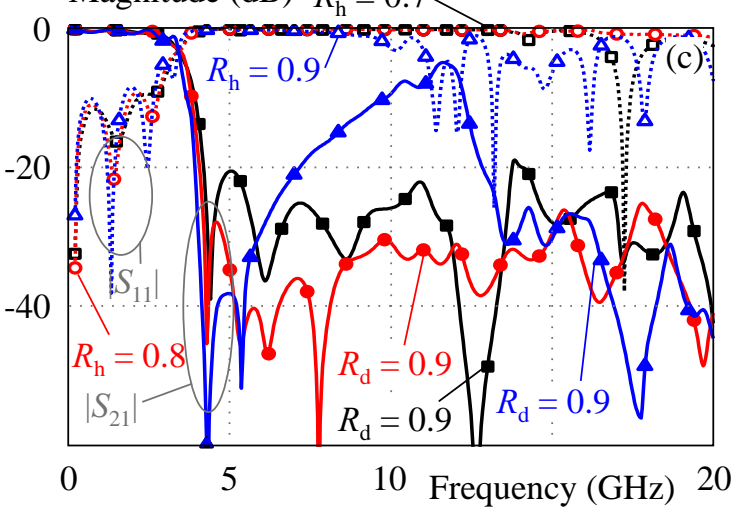

Magnitude (dB)

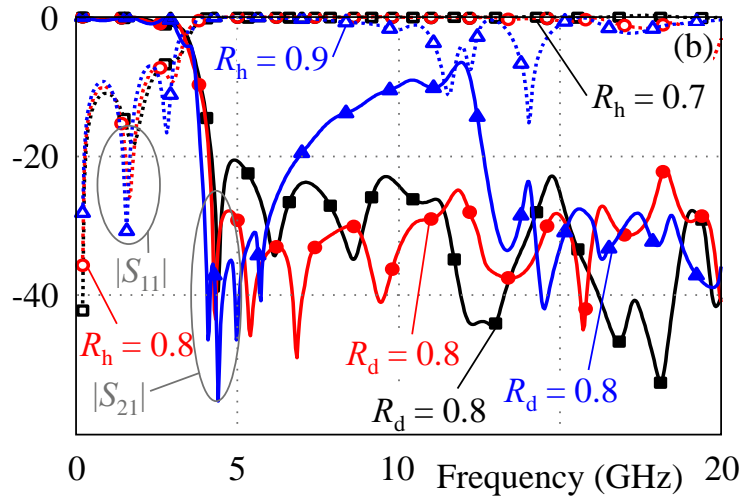

Magnitude (dB)

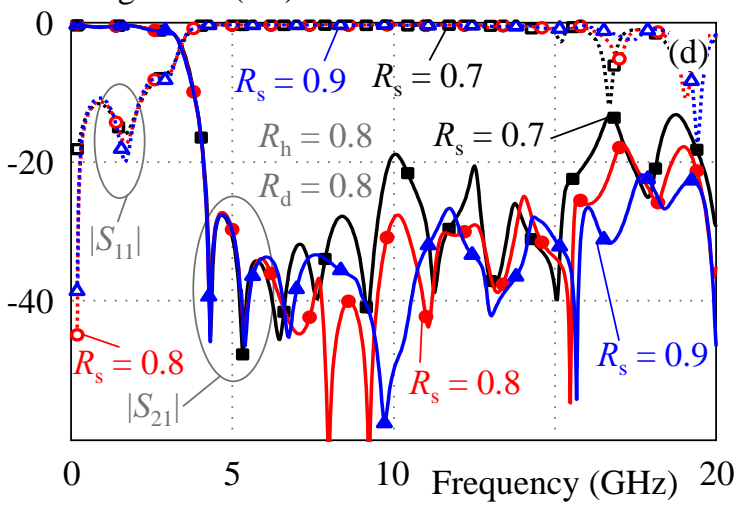

Fig. 3. Sweeping results for different separation(center to center)ratio $R_{s}$ and slotted length ratio $R_{\mathrm{h}}$, where the initial length(height)of the slot is $h_{1}=18.5 \mathrm{~mm}$ and the initial separation from the first to second slot is $d_{1}=6 \mathrm{~mm}$. (a)for $R_{\mathrm{h}}=0.7,0.8$ and 0.9 and $R_{\mathrm{d}}=$ 0.7.(b)for $R_{\mathrm{h}}=0.7,0.8$ and 0.9 and $R_{\mathrm{d}}=0.8$.(c)for $R_{\mathrm{h}}=0.7,0.8$ and 0.9 and $R_{\mathrm{d}}=0.9$.(d)for $R_{s}=0.7,0.8$ and 0.9 under $R_{\mathrm{h}}=0.8$ and $R_{\mathrm{d}}=0.8$

It is known that for the Yagi-Uda antenna design, the scale ratio, the slot dimensions and their environment need critically study for optimal electric performance. If the environment is not properly studied and scaled, such an antenna cannot work. These parameters are also very important in the microwave filter realization in this study. As a result, parameter sweeping study is carried out to have a deeper insight to the electric performance of the studied Yagi DGS filter. The simulation results are illustrated in Figs. 3(a)-(d), where the first slot has a constant length of $h_{1}=18.5 \mathrm{~mm}$ with the first separation of $d_{1}=$ $6 \mathrm{~mm}$ and a constant slot width of $s_{i}=1.5 \mathrm{~mm}$ for all cases shown in Figs. 3(a)-(c). In Fig. 3(a) the return and insertion losses have been plotted for the height scale ratio of $R_{\mathrm{h}}$ from 0.7 to 0.9 under a constant separation ratio of $R_{\mathrm{d}}=0.7$. It can be observed from Fig. 3(a) that for a smaller height ratio $R_{\mathrm{h}}$, wide suppression band can be achieved since this formulates the slot length varied greatly. Hence the shorter slots contribute to the high frequency suppressions. On the contrary, with the increasing of ratio $R_{\mathrm{h}}$ to a larger value, all slots tend to approach near the same length, thus the suppression at some high frequencies becomes poor. With increasing the separation ratio $R_{\mathrm{d}}$ to 0.8 and sweeping the height ratio $R_{\mathrm{h}}$ from 0.7 to 0.9 , similar response can be found as recorded in Fig. 3(b), where enhanced stopband suppression level can be observed as compared with the one shown in Fig. 3(a). Further increasing the separation ratio to $R_{\mathrm{d}}=0.9$, as shown in Fig. 3(c), one can see the suppression level in the stopband can be further increased under proper height ratios of $R_{\mathrm{h}}$, but this also yields increased circuit size. Consequently, the separation ratio $R_{\mathrm{d}}$ and height ratio
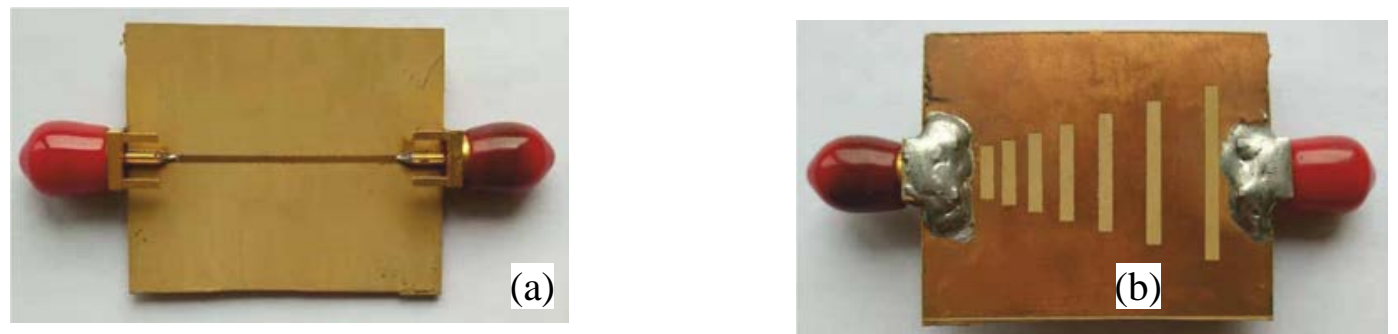

Fig. 4. Photograph of the developed prototype filter.(a) - front side, (b)- back side 
Table 1. Performance comparisons of the developed lowpass filter and some related publications

\begin{tabular}{|c|c|c|c|c|c|c|c|c|c|}
\hline \multirow[b]{2}{*}{$\begin{array}{l}\text { References } \\
\text { /Years }\end{array}$} & \multirow[b]{2}{*}{$\begin{array}{r}f_{c_{3} d B} \\
(\mathrm{GHz})\end{array}$} & \multirow{2}{*}{\multicolumn{2}{|c|}{$\begin{array}{l}\text { First attenuation } \\
\text { pole and level }\end{array}$}} & \multicolumn{2}{|c|}{$\begin{array}{l}\text { Stop-band range } \\
\text { suppression level }\end{array}$} & \multirow{2}{*}{$\begin{array}{l}\text { Filter } \\
\text { size }^{*} \\
\left(\mathrm{~mm}^{2}\right)\end{array}$} & \multicolumn{2}{|c|}{ Substrate } & \multirow[b]{2}{*}{ Notes } \\
\hline & & & & $\begin{array}{l}\text { supp } \\
(\mathrm{dB})\end{array}$ & ession level & & $\begin{array}{l}\varepsilon_{\mathrm{r}} \\
\mathrm{q}(-)\end{array}$ & $\begin{array}{c}h \\
(\mathrm{~mm})\end{array}$ & \\
\hline (19)/2014 & 1.8 & 35 & 2.9 & 20 & $2.8 \geq 10$ & $19.8 \times 15.4$ & 10.2 & 0.635 & Improved dumbbell \\
\hline$(20) / 2006$ & 3.75 & 30 & 4.35 & 20 & $4.3 \sim 15.8$ & - & 4.4 & 1.6 & Crossed dumbbell \\
\hline$(21) / 2016$ & 1.49 & 57.2 & 2.0 & 20 & $1.65 \sim 7.41$ & $40 \times 20$ & 3.38 & 0.813 & $\begin{array}{l}\text { Fan-shaped DGS } \\
\text { double radial stub }\end{array}$ \\
\hline$(22) / 2017$ & 4.0 & 32.8 & 6.7 & 20 & $4.3 \sim 20$ & $50 \times 14$ & 3.38 & 0.813 & Cross shaped DGS \\
\hline$(23) / 2017$ & 3.88 & 44.35 & 4.14 & 20 & $4.07 \sim 15$ & $35.25 \times 16.15$ & 2.55 & 1.5 & $\begin{array}{l}\text { Marbled microstrip } \\
\text { dumbbell-shaped DGS }\end{array}$ \\
\hline$(24) / 2015$ & 3.11 & 22.5 & 3.23 & 25 & $3.24 \sim 10.7$ & $20.02 \times 13.64$ & 10.2 & 1.27 & Interdigital DGS \\
\hline This work & 3.07 & 52 & 4.16 & 20 & $3.68 \sim>20$ & $23.64 \times 18.5$ & 9.6 & 0.8 & Yagi-DGS \\
\hline
\end{tabular}

$R_{\mathrm{h}}$ should be selected in such a way that some tradeoffs between the circuit size and the expected electric performance are required.

The slot width ratio $R_{s}$ is also studied here under constant values of $R_{\mathrm{d}}=0.8$ and $R_{\mathrm{h}}=0.8$. With $R_{s}$ sweeping, as presented in Fig. 3(d), results show that different from the height and separation with optimal ratios required, all slots should present uniform width to achieve higher suppressions within a wider stopband.

These parameter sweeping results gives some useful insights into the design and operation of this kind of Yagi shaped DGS, which can help enhance the design expediency and performances of this kind of filter.

A Yagi DGS lowpass filter is optimally designed based on above discussions. With electromagnetic optimizations, dimensions of the filter are found as: the first slot has a length of $h_{1}=18.5 \mathrm{~mm}$, the first separation is set to $d_{1}=6 \mathrm{~mm}$, the height or length ratio is $R_{\mathrm{h}}=0.74$, and the separation ratio is $R_{\mathrm{d}}=0.8$. Such a lowpass filter is realized on a microwave substrate mentioned above, which has a size of $23.635 \times 18.5 \mathrm{~mm}^{2}$.

\section{Measurements on the developed prototype filter}

The developed Yagi DGS lowpass filter prototype was fabricated to further validate its electric performance. Photographs of the developed demonstrator filter are shown in Fig. 4 with two SMA connectors attached at the two ends, where Fig. 4(a) is the front or top side of the filter and Fig. 4(b) shows the bottom/back side.

A vector network analyzer, N9918A from Agilent technologies, was utilized to experimentally characterize the electric performance of the developed filter. It can be observed from the measured and simulated results shown in Fig. 5 that in general, the passband insertion loss is less than $0.6 \mathrm{~dB}$ from $\mathrm{DC}$ to $3.07 \mathrm{GHz}$ with a $-3 \mathrm{~dB}$ cutoff frequency of $f_{c_{3} \mathrm{~dB}}=3.07 \mathrm{GHz}$. The developed lowpass filter has a stopband from 3.68 to over $20 \mathrm{GHz}$ with suppression level greater than $20 \mathrm{~dB}$ from measurements. The first transmission zero is located at $4.16 \mathrm{GHz}$ with suppression level over $52 \mathrm{~dB}$ from measurements, thus having a sharp transition from the passband to the stopband. The developed filter has an in-band group delay of about 0.4 ns. As a result of the open free space of the Yagi DGS patterns, the etched slots at some frequencies within the stop band act as electromagnetic emitter and thus emit electromagnetic waves into free space. This leads to some notches experienced in the measured return loss within the band of interest. In general, the measurements match with the simulations.

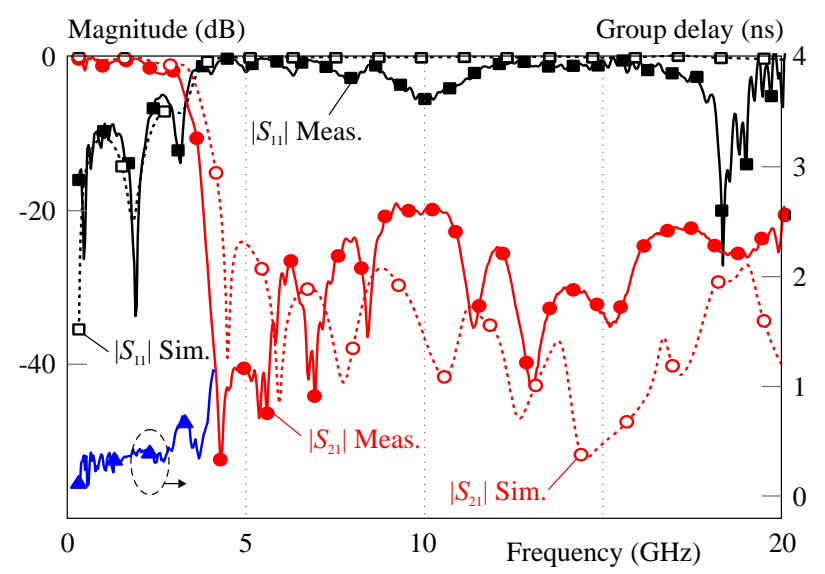

Fig. 5. Measurements and simulation results of the developed demonstrator filter

Further, Tab. 1 lists some published DGS based microwave lowpass filters and the developed lowpass filter in this study for performance comparisons. It can be observed from the table that, on the whole, the developed prototype demonstrator could characterize a comparatively high suppression level within a very wide stopband and a compact occupied circuit area. Moreover, the studied Yagi DGS is very simple in structure, as compared to other reported results presented in Table 1. 
Performance comparisons of the developed lowpass filter and some related publications.

\section{Conclusion}

A simple and compact Yagi DGS resonator has been introduced and discussed for microwave lowpass filter applications. The studied DGS exemplifies simple architecture, and can generate attenuation pole and reflection pole near the cutoff frequency. For microstrip lowpass filter demonstrations, results show an extensive stopband with high suppression level can be approached by cascading Yagi DGS slots. Experimental examinations on the developed prototype filter to authenticate the predications, consequently confirming the study of this contribution. It is envisaged that the presented Yagi DGS filter would be attractive for further integration with other microwave circuits, components or modules for system applications.

\section{REFERENCES}

[1] B.-A. Twumasi and J.-L. Li, "Numerical simulation study on bowtie antenna-based time reversal mirror for super-resolution target2 detection", Journal of Electrical Engineering, vol. 70, no. 3, pp. 236-243, June 2019.

[2] J. Zhou, J.-L. Li, C.-G. Sun, H. Li, and.-S. Gao, "A novel microstrip diplexer based on coupled line", Electromagnetics, vol. 38, no. 2, pp. 187-95, Mar. 2018.

[3] B.-A. Twumasi, J.-L. Li, and L. Xia, "A multi-coupled line based microstrip diplexer with high figure-of-merit", Electromagnetics, vol. 39, no. 7, pp. 465-472, August 2019.

[4] Y.-M. Zhang, S. Zhang, J.-L. Li, and G. Pedersen, "A dual-polarized linear antenna array with improved isolation using a slotline-based 180 hybrid for full-duplex applications", IEEE Antennas and Wireless Propagation Letters, vol. 18, no. 2, pp. 348-352, February 2019

[5] M. Yao, J.-L. Li, L. Xia, and S. Gao, "Wide dual-band dual-circularly polarized holographic metasurface", Journal of Physics D: Applied Physics, vol. 52, no. 42, pp. 425001(11pp), Oct. 2019.

[6] J.-N. Sun, J.-L. Li, and L. Xia, "A dual-polarized magneto-electric dipole antenna for application to N77/N78 band", IEEE Access, vol. 7, iss. 1, pp. 161708-161715, November 2019.

[7] C.-G. Sun, J.-L. Li, S.-Y. Yin, and S. Gao, "Broadband branch-line balun with good isolation and impedance matching", Electromagnetics, vol. 38, no. 5, pp. 273-282, May 2018.

[8] B.-A. Twumasi and J.-L. Li, "A compact, wideband branch-line balun with small magnitude and phase imbalances", Electromagnetics, vol. 39, no. 1, pp. 30-40, February 2019.

[9] S.-Y. Yin, J.-L. Li, and.-S. Gao, "Compact dual-band five-way Wilkinson power divider", Electronics Letters, vol. 53, iss. 13, pp. 866-868, June 2017.

[10] J.-L. Li, H.-Z. Wang, J.-P. Wang, S.-S. Gao, X.-S. Yang, and W. Shao, "Miniaturized Wilkinson power dividers with harmonic suppressions", Electromagnetics, vol. 36, no. 3, pp. 157-166, May 2016.

[11] L. Xia, J.-L. Li, B. A. Twumasi, P. Liu, and S.-S. Gao, "Planar dual-band branch-line coupler with large frequency ratio", IEEE ACCESS, vol. 8, iss.1, pp. 33188-33195, February 2020.

[12] L. Xia, J.-L. Li and. Liu, "Dual frequency Wilkinson power divider with 45 phase shifts at the two bands", Electromagnetics, vol. 40, iss. 3, pp. 199-206, February 2020.

13] B.-A. Twumasi and J.-L. Li, "An equivalent circuit model of a rectangular bracket shaped DGS and its microwave filter applications", Journal of Electrical Engineering, vol. 70, no. 1, pp. 32-38, February 2019.

[14] W. Shao and J.-L. Li, "Accurate modeling of a patterned ground and its application to microwave filters", Applied Computational Electromagnetics Society Journal, vol. 27, no. 7, pp. 596-602, July 2012.

$15]$ D.-E. Isbell, "Log periodic dipole arrays", IEEE Transactions on Antennas and Propagation, vol. 8, no. 5, pp. 260-267, May 1960 .

16] N. Barbano, "Log periodic Yagi-Uda array", IEEE Transactions on Antennas and Propagation, vol. 14, no. 2, pp. 235-238, Mar. 1966.

[17] C.-K. Campbell, I. Traboulay, M.-S. Suthers, and H. Kneve, "Design of a stripline log-periodic dipole antenna", IEEE Transactions on Antennas and Propagation, vol. 25, no. 5, 718-721, Sept. 1977.

[18] W. Shao and J.-L. Li, "A compact log-periodic branch-line balun with an octave bandwidth", Journal of Electromagnetic Waves and Applications, vol. 25, no. 14-15, pp. 2033-2042, Sept. 2011.

[19] A. Boutejdar, "Design of broad-stop band lowpass filter using a novel quasi-Yagi-DGS- resonators and metal box-technique", Microwave and Optical Technology Letters, vol. 56, no. 3, pp. 523-528, Mar. 2014

20] H.-J. Chen, T.-H. Huang, C.-S. Chang, L.-S. Chen, N.-F. Wang, Y.-H. Wang, and P. Houng, "A novel cross-shape DGS applied to design ultra-wide stopband low-pass filters", IEEE Microwave and Wireless Components Letters, vol. 16, no. 5, pp. 252-254, May 2006.

21] A.-O. Ertay, M. Abbak, and S. Simsek, "An improved stopband and sharp roll off microstrip low pass filter with defected ground structures", International Journal of Microwave and Wireless Technology, vol. 8, no. 3, pp. 573-581, Mar. 2016.

[22] A. Boutejdar and S.-D. Bennani, "Improvement of low pass and band pass filter characteristics using cross-DGS-resonators, logarithmic series and coupling matrix methods", International Conference on Wireless Technology, Embedded and Intelligent Systems (WITS), Fez, Morocco, 19 April -20 April 2017, pp. 1-6.

23] P. Zhang and M. Li, "A novel sharp roll-off microstrip lowpass filter with improved stopband and compact size using dual-plane structure", Microwave and Optical Technology Letters, vol. 58, no. 5, pp. 1085-1088, May 2016.

[24] S. Liu, J. Xu and Z. Xu, "Sharp roll-off lowpass filter using interdigital DGS slot", Electronics Letters, vol. 51, no. 17, pp. 1343-1345, August 2015.

Received 19 April 2020 